\title{
Astrophysics and Space Science introduces article numbering
}

\author{
Ramon Khanna $^{1} \cdot$ Elias Brinks $^{2} \cdot$ Jeremy Mould $^{3}$
}

Received: 4 March 2015 / Accepted: 4 March 2015 / Published online: 24 March 2015

(C) Springer Science+Business Media Dordrecht 2015

Bibliographic information of articles published in physics and astronomy journals is changing rapidly towards a system in which articles are referenced individually rather than using page numbering within issues and volumes of a given journal. This way any delay in assigning the final citation information to an article is avoided.

Beginning with Volume 357 (May/June 2015) the journal Astrophysics and Space Science adopts such a system. Instead of a continuous pagination of articles throughout a volume, each article will have an internal pagination beginning with page 1 . The identification of an article within a volume by its first page number will be replaced by identification by an article citation-id. The bibliographic information (the "reference") shown in the header on the first page of an article will be built as follows:

Astrophys Space Sci ([publication year]) [volume number]:[article citation-id] DOI [DOI]

(an example is the bibliographic information in the header of this editorial).

With this change, papers will be available with their full and final bibliographical information as soon as they are published online and will fill up the currently open issue until its closure date. The journal will therefore stop publishing articles in Springer's Online First ${ }^{\mathrm{TM}}$ modus. The time from acceptance to online publication of an article is not affected by this change. The final citation of an article will be of the form:

\footnotetext{
$凶$ R. Khanna

Ramon.Khanna@springer.com

Springer, Heidelberg, Germany

2 University of Hertfordshire, Hatfield, UK

3 Swinburne University of Technology, Hawthorn, Australia
}

Khanna, R, Brinks, E, Mould, J (2015) Astrophysics and Space Science Introduces Article Numbering, Astrophys Space Sci, 357:1, DOI 10.1007/s 10509-015-2225-7

We believe this change will make a step towards a simpler system of bibliographic information within the framework of short online publication times of individual articles. 